\title{
Brain death in low-income countries: a report from Malawi
}

\author{
Meghan Prin ${ }^{1,2}$ (D), Caroline Quinsey ${ }^{3}$, Clement Kadyaudzu ${ }^{4}$, \\ Eldad Hadar ${ }^{5}$ and Anthony Charles ${ }^{6}$
}

\footnotetext{
'Assistant Professor, Department of Anesthesiology, Baylor College of Medicine, Houston, TX, USA

${ }^{2}$ Department of Obstetrics \& Gynecology, Baylor College of Medicine, Houston, TX, USA

${ }^{3}$ Assistant Professor, Department of Neurosurgery, University of North Carolina at Chapel Hill, Chapel Hill, NC, USA

${ }^{4}$ Clinical Officer, Kamuzu Central Hospital, Lilongwe, Malawi
}

Tropical Doctor 2019, Vol. 49(2) 107-112 


\begin{abstract}
Most low-income nations have no practice guidelines for brain death; data describing brain death in these regions is absent. Our retrospective study describes the prevalence of brain death among patients treated in an intensive care unit (ICU) at a referral hospital in Malawi. The primary outcome was designation of brain death in the medical chart. Of 449 ICU patients included for analysis between September 2016 and May 2018, 43 (9.6\%) were diagnosed with brain death during the ICU admission. The most common diagnostic reasons for admission among these patients were trauma (49\%), malaria $(16 \%)$ and postoperative monitoring after general abdominal surgery $(19 \%)$. All patients diagnosed with brain death were declared dead in the hospital, after cardiac death. In conclusion, the incidence of brain death in a Malawi ICU is substantially higher than that seen in high-income ICU settings. Brain death is not treated as clinical death in Malawi.
\end{abstract}

\title{
Keywords
}

Brain death, global health, low-resource, critical care

\section{Introduction}

The concept of brain death first emerged in the 1950s, driven by advances in critical care medicine such as cardiopulmonary resuscitation and mechanical ventilation. In countries where it is an accepted concept, a patient determined to be brain dead is legally and clinically dead with an irreversible loss of all brain function. ${ }^{1}$ While the precise criteria for this diagnosis have changed over time in the evolving context of critical care medicine and advanced neurologic diagnostic techniques, they remain based on a clinical neurological examination. ${ }^{1}$ Practice guidelines were published by the American Academy of Neurology in $1995^{2}$ and updated in 2010. ${ }^{1}$ Despite these definitions and guidelines, brain death lacks global acceptance and there are substantial differences in the application of this diagnosis worldwide. ${ }^{3}$

Most low-income countries (LIC) have no legal nor clinical practice guidelines for brain death. ${ }^{3}$ The reasons cited for a lack of guidelines by LIC respondents in one large global survey included: (1) lack of physician expertise; and (2) uncertainties about the concept. ${ }^{3}$ There is also sociocultural and/or religious resistance to this concept in many nations. ${ }^{4-6}$ Diagnosing brain death is a first step to addressing the futility of further intensive or invasive care and might influence triage decisions in low-resource areas. This may be especially relevant in LICs, where hospital and intensive care unit (ICU) beds are very scarce. Reframing the concept of brain death in the context of organ failure may motivate healthcare providers in LICs to perform a relevant neurologic examination. Increased identification of brain death may be disproportionately beneficial to LIC healthcare systems where specialists in neurology, neurological surgery and critical care are often absent. However, prospective research on this topic in lowincome settings is essentially absent. The objective of our study was to describe the prevalence of brain death among patients treated in an ICU at a central referral hospital in Malawi.

\section{Methods}

Ours was a retrospective, single-centre observational study of patients admitted to the ICU of Kamuzu Central Hospital $(\mathrm{KCH})$, a central referral hospital in Lilongwe, Malawi, from September 2016 to May 2018. Malawi is a small landlocked country in southeast Africa bordered by Zambia, Tanzania and Mozambique (Figure 1). It is one of the poorest countries in the world with a life expectancy of 63.8 years and a Human Development Index rank of 170 out of 187 countries. $^{7}$

$\mathrm{KCH}$ is a 700-bed referral hospital in the central region of Malawi with a catchment area of approximately 6 million. It has a computed tomography (CT) scanner and an ICU with five ventilator-equipped beds. The ICU offers continuous non-invasive vital sign monitoring (i.e. no arterial line equipment) and can provide intravenous vasopressor or sedative infusions. The nursing ratio is $1: 1$ and clinical care in the unit is directed by clinical officers in Anesthesiology. ${ }^{8}$ No consultant physicians are on staff with expertise in intensive care medicine or neurology. A consultant in neurological surgery is available, but there is currently no equipment for intracranial pressure monitoring nor surgical procedures other than exploratory burr-holes.

The hospital also has four high-dependency units (HDUs), which serve as both 'step-up' and 'step-down' areas for patients developing or recovering from critical illness, respectively. ${ }^{9}$ These units offer continuous vital sign monitoring (viz. pulse oximetry and electrocardiogram) and a higher nurse-to-patient ratio than that of the general ward, but do not offer mechanical ventilation.

The data used for analysis were taken from an existing database, which was collected prospectively by data clerks trained specifically in ICU data abstraction. The primary outcome of our study was the designation of brain death in the medical chart by the overseeing clinical officers. While there is no specific policy in 


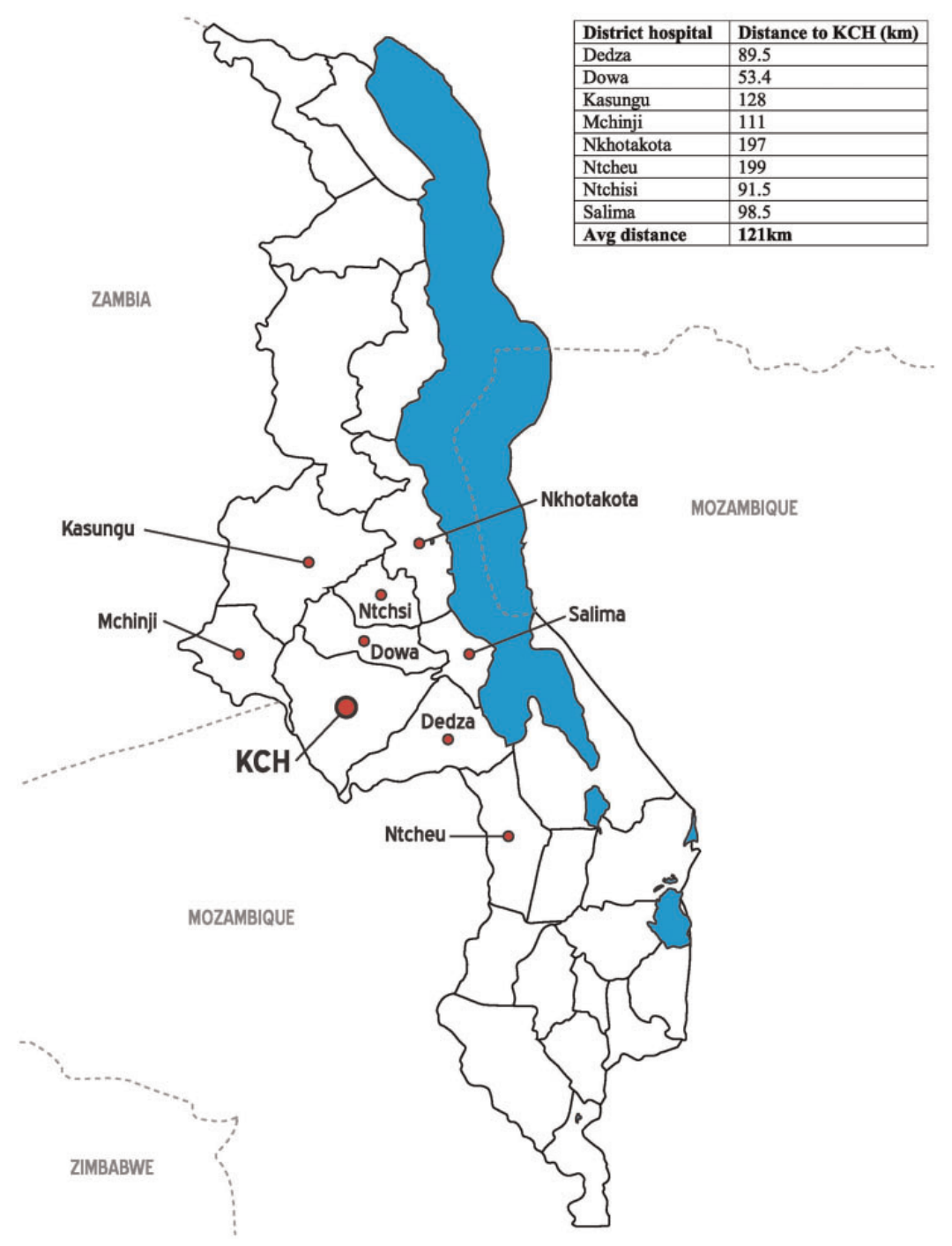

Figure I. Map of Malawi with notation for study site, Kamuzu Central Hospital (KCH).

diagnosing brain death at $\mathrm{KCH}$, all providers include an assessment of lack of brain stem reflexes and lack of respiratory effort when pausing the mechanical ventilator. Further investigations such as arterial blood gas analysis and/or advanced imaging are not available at this central referral hospital nor at any district hospitals, consistent with most other hospitals in the region.

The only inclusion criterion was the designation of a brain death diagnosis in the medical chart by the overseeing clinical officer, at any time during the course of ICU stay. The designation and record of brain death varied because brain death does not exist as a legal entity in Malawi. ICU staffing experience varies: notably, some staff check for brain death daily while others do not. Some staff record their examinations in the medical chart while others do not. Furthermore, exact times are not recorded for diagnoses in the medical chart at this study hospital and this information was not available.

We excluded ICU readmissions from analysis to avoid double-counting patients with brain death who may have been discharged from the ICU and readmitted from various hospital areas. Clinical data points collected included date of hospital admission, location before ICU admission (e.g. emergency room, operating theatre), vital signs and laboratory measurements (e.g. serum sodium, platelets) at admission to ICU, treatments utilised during the ICU stay (e.g. mechanical ventilation, tracheostomy, CT scanning), human immunodeficiency virus serostatus, malaria point-ofcare testing, the hospital location to which patients were discharged, the length of stay in the ICU (in calendar days), and the date of hospital discharge or death. 
We described the patients diagnosed with brain death and compared clinical characteristics between these patients and those without a brain death diagnosis.

Continuous variables were described using the median and interquartile range (IQR) and categorical variables using the frequency and percentage, unless otherwise noted. To compare patients with and without brain death, we used the $\chi^{2}$ test for categorical variables and Student's t-test for continuous variables. Statistical significance was set at $P<0.05$ for all analyses, which were conducted using Stata/SE 14.2 (StataCorps, College Station, TX, USA). Ethical approval for the study was provided by the National Health Sciences Research Council of Malawi and the Institutional
Review Boards of Columbia University and the University of North Carolina at Chapel Hill.

\section{Results}

During the study period, 456 patients were admitted to the study ICU and, after excluding readmissions, there were 449 patients included in the analysis. Of these, 43 $(9.6 \%)$ were diagnosed with brain death during ICU admission. The timing of this diagnosis was unavailable. Demographic data are shown in Table 1.

Among patients who were diagnosed with brain death at some point during their ICU stay, the most common reason for ICU admission was trauma (49\%)

Table I. Clinical characteristics and outcomes for patients admitted to the ICU of Kamuzu Central Hospital in Lilongwe, Malawi, from September 2016 through May 2018.

\begin{tabular}{|c|c|c|c|}
\hline & $\begin{array}{l}\text { Brain death } \\
(n=43)\end{array}$ & $\begin{array}{l}\text { No brain death } \\
(n=406)\end{array}$ & $P$ value \\
\hline Male (n (\%)) & $27(64)$ & $178(44)$ & 0.018 \\
\hline Age (median (IQR)) & $29(18-40)$ & $27(19-38)$ & 0.488 \\
\hline Admission secondary to trauma (n (\%)) & $21(49)$ & $96(24)$ & $<0.001$ \\
\hline Location before ICU admission (n (\%)) & & & $<0.001$ \\
\hline Operating theatre & $8(19)$ & $186(47)$ & \\
\hline HDU & II (26) & $97(25)$ & \\
\hline Ward & $6(14)$ & $38(10)$ & \\
\hline Emergency Room & $17(40)$ & $59(15)$ & \\
\hline Outside hospital & I (2) & $12(3)$ & \\
\hline \multicolumn{4}{|l|}{ Vital signs at ICU admission } \\
\hline Heart rate (bpm) (median (IQR)) & $119(94-131)$ & $120(|02-| 4 \mid)$ & 0.229 \\
\hline Mean arterial pressure $(\mathrm{mmHg})($ median $(\mathrm{IQR}))$ & $85(65-104)$ & $87(7 \mid-103)$ & 0.229 \\
\hline Temperature $\left({ }^{\circ} \mathrm{C}\right)$ (median $\left.(\mathrm{IQR})\right)$ & $36(35-37)$ & $36(34-37)$ & 0.824 \\
\hline Oxygen saturation (median (IQR)) & $98(95-100)$ & $97(92-99)$ & 0.803 \\
\hline CT scan of the head performed before ICU admission (n (\%)) & $7(16)$ & $40(10)$ & 0.191 \\
\hline CT scan of the head during ICU stay (n (\%)) & $4(9)$ & $23(6)$ & 0.340 \\
\hline \multicolumn{4}{|l|}{ Laboratory values at ICU admission } \\
\hline Platelet count (cells $\left.\times 10^{9} / \mathrm{L}\right)($ median $(\mathrm{IQR}))$ & $130(72-191)$ & $154(99-255)$ & 0.094 \\
\hline Sodium (mEq/L)* (median (IQR)) & |43 (|39-149) & $143(139-147)$ & 0.436 \\
\hline Creatinine $(\mu \mathrm{mol} / \mathrm{L})(\operatorname{median}(\mathrm{IQR}))$ & $91.9(58.3-210.4)$ & $90.2(58.3-216.6)$ & 0.370 \\
\hline HIV status (positive) (median (IQR)) & $5(15)$ & $34(10)$ & 0.449 \\
\hline Malaria status (positive) (median (IQR)) & II (28) & $44(15)$ & 0.030 \\
\hline Provision of mechanical ventilation (n (\%)) & $43(100)$ & $390(97)$ & 0.232 \\
\hline Days of mechanical ventilation (median (IQR)) & $3(2-8)$ & $2(I-5)$ & 0.863 \\
\hline Provision of tracheostomy (n (\%)) & $6(14)$ & $46(12)$ & 0.639 \\
\hline Time to tracheostomy (days) (median (IQR)) & $8(0-12)$ & $10(7-13)$ & 0.226 \\
\hline ICU length of stay (days) (median (IQR)) & $3(2-8)$ & $3(I-5)$ & 0.796 \\
\hline Hospital length of stay (days) (median (IQR)) & $5(3-8)$ & $8(3-17)$ & 0.007 \\
\hline Hospital mortality (n (\%)) & $43(100)$ & $219(57)$ & $<0.001$ \\
\hline
\end{tabular}

*Milliequivalents per litre. 
Table 2. Reason for ICU admission among patients who were diagnosed with brain death in the ICU.

$\begin{array}{lc}\text { Reason for admission } & \mathrm{n}(\%) \\ \text { Head injury } & 18(42) \\ \text { General trauma } & 3(7) \\ \text { Malaria } & 7(16) \\ \text { Meningitis } & 1(2) \\ \text { Respiratory failure } & 4(9) \\ \text { Eclampsia } & 2(5) \\ \text { Postoperative monitoring, bowel surgery } & 8(19) \\ \text { Total } & 43(100)\end{array}$

followed by malaria (16\%) and postoperative monitoring after general abdominal surgery because of undifferentiated intraoperative complications (19\%). (Table 2) Clinical details are shown in Table 1. All patients diagnosed with brain death were ultimately declared dead in the hospital, subsequent to cardiac death.

\section{Discussion}

Our study demonstrates that in a critically ill cohort of African patients in a low-resource setting, $9.6 \%$ of patients were diagnosed with brain death during ICU stay. This is substantially higher than the incidence recorded in ICUs in high-income settings. ${ }^{10,11}$ All the patients diagnosed with brain death remained in the ICU or a HDU until cardiac death. There is thus no recognition of brain death as clinical death in Malawi. These data highlight the need to investigate more thoroughly the incidence of brain death and understand the treatment options of this patient population in a lowincome African setting.

A multitude of studies describe a striking variation in the application of evidence-based guidelines for the diagnosis of brain death. ${ }^{3,6,12}$ While international standardisation of clinical criteria to diagnose brain death has been advocated as a solution for developing healthcare systems, ${ }^{12}$ sociocultural resistance to this concept is relevant and must be taken into account in order to develop realistic strategies to improving healthcare utilisation. In the context of ICU medicine, the ethical imperative to do no harm is weighed daily against the need to do the most good for the population at large. Healthcare rationing is perhaps never as difficult as it is in the assessment of the critically ill, when the timing of interventions may significantly impact outcomes. In general, ICU care should logically be reserved for those who are most likely to benefit. Patients who are 'too well' should not be admitted to ICU care, nor should those who are 'too sick' to benefit. A recent medical editorial from
Malawi emphasised this ethical imperative. ${ }^{13}$ Patients diagnosed with brain death clearly fall into the 'too sick' category; yet they account for a sizeable proportion of the ICU population in this study. Addressing this ethical conundrum requires broad input from the populace, government leaders, healthcare experts, cultural and religious leaders, and ethicists.

One possible way to address the sociocultural concerns or discomfort regarding brain death may be to reframe the concept, from brain death to irreversible brain failure. This is not just semantics. In critical care, clinicians frequently discuss organ failure. Moving away from the word 'death' may encourage clinicians to perform neurologic examinations to assess brain failure and also help them appreciate that brain failure is an end-stage consequence of brain injury (an all too common entity in LICs). While some organs may be supported by pharmacotherapy or mechanical devices, the ability to support the brain in any setting is limited. Performing a neurologic examination to assess for brain failure may appear quite different to clinicians than performing an examination to confirm brain death. After ascertaining that a patient meets criteria for brain failure, clinicians may feel more comfortable prognosticating with certainty and conducting family discussions. In order to test the effectiveness of this suggested change in nomenclature on the rates of brain death diagnosis and accompanying ICU utilisation, follow-up studies are required. These studies should investigate the qualitative effects of this nomenclature change on staff and family perceptions of brain death and the quantitative effects of this nomenclature on the detection of brain death and ICU utilisation.

Even though brain death is not necessarily recognised as clinical death at our study hospital in Malawi, the record of this diagnosis in the patient chart remains notable. This implies that overseeing clinicians have a conceptual acceptance of this diagnosis, recognise signs of brain death and value this diagnosis enough to record it. The transition from diagnosing and recording brain death to acting on it (e.g. withdrawing care) may be more nuanced in certain regions of the world than others. ${ }^{14}$ However, the withdrawal of resources from patients with brain death may allow the hospital to care for other patients with a better chance of recovery. It is on this trade-off that patients, families, and healthcare providers in LICs with low-resource healthcare systems must reflect. In a recent international survey designed to assess international variation in brain death definitions, only $10 \%$ of respondents were from LICs. Respondents from these countries were least likely to have recent experience in diagnosing brain death or to report institutional protocols for brain death, but most likely to have greater experience in rationing available healthcare. ${ }^{3}$ Even in a setting where brain death is 
accepted socially and culturally, this different experience may be seen as disempowering. Reframing brain death as brain failure could, however, empower clinicians by providing more familiar terminology, which may also better equip them in their medical recommendations.

After overcoming sociocultural barriers to the concept of brain death, the determination of brain death or failure will remain challenging in LICs because of limitations in diagnostic technology, particularly the lack of imaging equipment and arterial blood gas analysers to conduct apnoea tests. Recognition of this reality should not preclude quality research in this arena; rather, it should highlight the need for a consensus definition of brain death that is feasible to apply in low-resource environments. The severity of this situation in Malawi is evidenced by the fact that only a small proportion of patients with brain death received a CT scan of the head during their hospital stay. Our proposal to reframe this concept is not meant to oversimplify these challenges, but rather to provide a new way in which to approach them.

Our study has several limitations. First, the study hospital has no formal criteria for the diagnosis of brain death. Therefore, it is unlikely that patients with documented brain death meet a single set of criteria. Second, because this was a secondary analysis of an existing dataset, we were unable to validate the diagnosis of brain death, or to precisely monitor individual clinicians' brain death examinations, or to capture the timing of brain death within the hospital stay. Finally, the scarcity of ICU beds in this low-resource hospital means that many patients in need of ICU or HDU care cannot access it. For this reason, we may not have captured all critically ill patients at risk of brain death. Considering these limitations, our reported prevalence of brain death among critically ill patients in Malawi is approximate but probably an underestimate, though more precise investigations are needed.

\section{Acknowledgements}

The authors thank Kathryn Pietrosimone, PhD, Scientific Research Manager at the Lineberger Comprehensive Cancer Center, University of North Carolina at Chapel Hill, for her input and edits to the manuscript.

\section{Declaration of conflicting interests}

The author(s) declared no potential conflicts of interest with respect to the research, authorship, and/or publication of this article.

\section{Funding}

The author(s) disclosed receipt of the following financial support for the research, authorship, and/or publication of this article:
This work was funded in part by the Fogarty International Postdoctoral Clinical Research Fellowship, to Dr. Prin, through the University of North Carolina at Chapel Hill.

\section{ORCID iD}

Meghan Prin (D) http://orcid.org/0000-0002-2886-4666

\section{References}

1. Wijdicks EF, Varelas PN, Gronseth GS, et al. Evidencebased guideline update: determining brain death in adults: report of the Quality Standards Subcommittee of the American Academy of Neurology. Neurology 2010; 74: 1911-1918.

2. Wijdicks EF. Determining brain death in adults. Neurology 1995; 45: 1003-1011.

3. Wahlster S, Wijdicks EF, Patel PV, et al. Brain death declaration: Practices and perceptions worldwide. Neurology 2015; 84: 1870-1879.

4. Yang Q and Miller G. East-West differences in perception of brain death. Review of history, current understandings, and directions for future research. $J$ Bioeth Inq 2015; 12: 211-225.

5. Arbour R, AlGhamdi HM and Peters L. Islam, brain death, and transplantation: culture, faith, and jurisprudence. AACN Adv Crit Care 2012; 23: 381-394.

6. Ding ZY, Zhang Q, Wu JW, et al. A Comparison of Brain Death Criteria between China and the United States. Chin Med J (Engl) 2015; 128: 2896-2901.

7. United Nations Development Programme. Human Development Reports. New York, NY: UNDP, 2016. Available at: www.hdr.undp.org/en.

8. Ashengo T, Skeels A, Hurwitz EJH, et al. Bridging the human resource gap in surgical and anesthesia care in low-resource countries: a review of the task sharing literature. Hum Resour Health 2017; 15: 77.

9. Prin M and Wunsch H. The role of stepdown beds in hospital care. Am J Respir Crit Care Med 2014; 190: 1210-1216.

10. Staworn D, Lewison L, Marks J, et al. Brain death in pediatric intensive care unit patients: incidence, primary diagnosis, and the clinical occurrence of Turner's triad. Crit Care Med 1994; 22: 1301-1305.

11. Escudero D, Valentin MO, Escalante JL, et al. Intensive care practices in brain death diagnosis and organ donation. Anaesthesia 2015; 70: 1130-1139.

12. Smith M. Brain death: time for an international consensus. Br J Anaesth 2012; 108(Suppl. 1): i6-9.

13. Manda-Taylor L, Mndolo S and Baker T. Critical care in Malawi: the ethics of beneficence and justice. Malawi Med J 2017; 29: 268-271.

14. Waweru-Siika W, Clement ME, Lukoko L, et al. Brain death determination: the imperative for policy and legal initiatives in Sub-Saharan Africa. Glob Public Health 2017; 12: 589-600. 